\title{
Comparação de procedimentos de seleção para produção de grãos em populações de soja
}

\author{
Comparison of selection procedures for grain production in soybean populations
}

\author{
Edésio Fialho dos Reis ${ }^{1}$ Múcio Silva Reis ${ }^{2}$ Cosme Damião Cruz $^{3}$ Tuneo Sediyama $^{4}$
}

\section{RESUMO}

Este trabalho teve como objetivos predizer o ganho genético, para produção de grãos, advindo de diferentes procedimentos de seleção e indicar, entre as populações estudadas, a mais promissora quanto à expectativa de ganhos. Quatro estratégias de seleção foram utilizadas na comparação de ganhos: seleção entre e dentro de famílias; seleção combinada e seleção individual para produção de grãos; e, também, para a seleção livre de pesos e de parâmetros, o número de dias e altura da planta por ocasião da maturação. Os indivíduos selecionados encontravamse na geração $F_{5}$ e foram conduzidos e observados em campo no esquema cultivares-padrão intercalados às linhas segregantes, no ano agrícola 1996/97, em Viçosa, Minas Gerais. Os resultados indicaram que, considerando a família no processo seletivo, a estratégia de seleção combinada foi a mais promissora. Entretanto, a estratégia de seleção individual deteve maior expectativa de progresso genético entre as quatro estratégias. Quanto aos cruzamentos, o cruzamento entre os progenitores CEPS 89-26 e FTCristalina foi o que apresentou maior expectativa de ganho genético.

Palavras-chave: Glycine $\max ($ L.) Merrill, herdabilidades, predição de ganhos.

\section{ABSTRACT}

This study aimed to predict the genetic gain for grain production by applying different selection procedures and to choose, among the studied populations, the most promising one regarding gain expectation. Four selection strategies were used: selection among and within families; combined selection and individual selection for grain production; as well as for selection free from weights and parameters, number of days and plant height at maturation. Generation $F_{5}$ individuals were selected, placed and observed in the field following the design standard-cultivars among the segregating lines, during the agricultural year of 1996/ 1997, in Vicosa, Minas Gerais. The results indicated that, considering the family in the selective process, combined selection was the most promising strategy. However, individual selection showed the highest expectation of genetic gain among the four strategies. The cross between the parents CEPS 89-26 and FTCristalina presented the highest expectation of genetic gain.

Key words: Glyine max (L.) Merrill, heritability, gain prediction.

\section{INTRODUÇÃO}

O progresso genético direcionado em qualquer espécie está associado à existência de variabilidade genética, à seleção natural e/ou artificial e ao ajuste dos genótipos aos ambientes existentes. Comprovada a presença da variabilidade genética e sobretudo o valor desta em relação à variação não-genética, a seleção assume grande importância no progresso genético. A seleção objetiva acumular alelos favoráveis à característica de interesse em determinada população e é um processo vinculado a uma constante e permanente renovação.

A possibilidade do melhorista predizer o ganho esperado pela seleção, antes mesmo que ela seja realizada, é, sem dúvida, uma das maiores contribuições da genética quantitativa (RAMALHO \&

\footnotetext{
${ }^{1}$ Engenheiro Agrônomo, Doutor, Prof. Adjunto, Campus Avançado de Jataí, Universidade Federal de Goiás, 75800-000, Jataí, GO. E-mail: edesio@ jatai.ufg.br. Autor para correspondência

${ }^{2}$ Engenheiro Agrônomo, Doutor, Professor Titular, Departamento de Fitotecnia, Universidade Federal de Viçosa (UFV). 36571-000, Viçosa, MG. Bolsista CNPq.

${ }^{3}$ Engenheiro Agrônomo, Doutor, Professor Titular, Departamento de Fitotecnia, UFV. Bolsista CNPq.

${ }^{4}$ Engenheiro Agrônomo, PhD, Professor Titular, Departamento de Fitotecnia, UFV. Bolsista CNPq.
} 
VENCOVSKY, 1978; HALLAUER \& MIRANDA FILHO, 1981). Para CRUZ \& REGAZZI (1997), de acordo com a estratégia de seleção e o ganho que ela proporcionará, pode-se orientar, de maneira mais efetiva, um programa de melhoramento, bem como predizer o sucesso do esquema seletivo adotado, decidindo, com bases científicas, quais esquemas podem resultar em maior ganho genético.

Os progressos genéticos referem-se às alterações observadas nas características de interesse, durante um ciclo de seleção, com a recombinação e multiplicação das unidades selecionadas. Tais modificações ocorrerão em magnitude e sentido variados, dependendo da estratégia e dos critérios de seleção adotados. Assim, uma das atribuições mais importantes do melhorista de plantas é identificar critérios de seleção capazes de promover alterações, no sentido desejado, nas características de interesse dentro de um programa de melhoramento.

Vários são os procedimentos utilizados pelo melhorista para identificar os genótipos superiores numa população. Alguns levam em conta o comportamento do indivíduo, enquanto outros se fundamentam, primeiramente, no desempenho da família e, secundariamente, na superioridade relativa dos indivíduos dentro da família. Há, também, a estratégia que utiliza, simultaneamente, as informações do indivíduo e de seus parentes, chamada de seleção combinada, sendo sempre superior aos métodos de seleção individual, de famílias e entre e dentro de famílias (FALCONER, 1987). A seleção combinada proporciona resposta superior ou, no mínimo, equivalente à seleção de famílias ou à seleção massal (LUSH, 1964), e a resposta a seleção pode ser maximizada se toda informação obtida nos parentes for também utilizada no processo seletivo (WEBER, 1982).

A seleção em gerações iniciais, principalmente individual, está na dependência da avaliação de cada indivíduo na parcela, razão por que se deve utilizar maior espaçamento para facilitar as mensurações; com isso, a seleção pode não ser favorável àqueles genótipos desejáveis (JENNINGS \& HERRARA, 1968; KHALIFA \& QUALSET, 1975).

O objetivo deste trabalho foi comparar diferentes procedimentos de seleção quanto à predição de ganhos para dados corrigidos pela variação de linhas homozigóticas (cultivares-padrão) e não corrigidos, com referência a produção de grãos, assim como comparar o comportamento dos diferentes cruzamentos utilizados, quanto aos procedimentos de seleção.

\section{MATERIAL E MÉTODOS}

Foram utilizados três cruzamentos, os quais se encontravam na geração $\mathrm{F}$ : CR1: 'CEPS 77-16' $\mathrm{x}$ 'Doko RC'; CR2: 'CEPS 89'-26' x 'IAC-8' e CR3: 'CEPS 89-26' x 'FT-Cristalina'. As gerações anteriores foram conduzidas em " bulk" por pesquisadores do Programa de Melhoramento de Soja do Departamento de Fitotecnia da Universidade Federal de Viçosa (UFV). O experimento foi conduzido em Viçosa, Minas Gerais, no ano agrícola 1996/97.

A constituição experimental no campo foi a de linhas segregantes com cultivares-padrão intercalados à semelhança de ensaios clássicos de análise de geração, tais como $\mathrm{P}_{1}, \mathrm{P}_{2}, \mathrm{~F}_{1}$ e $\mathrm{F}_{2}$. Nesse caso a população segregante é representada por $\mathrm{F}_{5}$ ao invés de $\mathrm{F}_{2} \mathrm{e}$ as populações uniformes (em homozigose) são representadas pelos cultivares-padrão (genitores), ao invés de $\mathrm{P}_{1}, \mathrm{P}_{2}$ e $\mathrm{F}_{1}$. $\mathrm{O}$ arranjo no campo foi feito de forma que a cada cinco linhas um dos cultivares-padrão era inserido, começando sempre por um deles. Foram utilizados como cultivares-padrão um dos parentais e o cultivar BR 16, este como substituto das linhagens CEPS 77-16 e CEPS 89-26, devido à não-disponibilidade de sementes dessas linhagens e ao fato de o cultivar BR 16 apresentar características agronômicas semelhantes às das linhagens. Cada parcela foi constituída de uma fileira de $1,0 \mathrm{~m}$ de comprimento, no espaçamento de $0,70 \mathrm{~m}$ entre fileiras, sendo utilizadas 30 sementes na fileira. Aos 15 a 20 dias após a emergência, foi feito o desbaste, deixando-se 15 plantas por parcela.

Para efeito de avaliação, foram etiquetadas seis plantas competitivas dentro de cada parcela, sendo isso feito antes do florescimento, para que não houvesse tendenciosidade na escolha das plantas a serem avaliadas, o que, se acontecesse, levaria a uma seleção para ciclo. Foram avaliadas as plantas etiquetadas previamente em cada parcela e os seguintes caracteres foram utilizados: número de dias para maturação - contados a partir da semeadura até que $95 \%$ das vagens atingissem a coloração típica de maturação; altura da planta na maturação - altura, em centímetros, do nível do solo até o último nó da haste principal, por ocasião da maturação; produção de grãos - peso total das sementes de cada planta, em gramas.

\section{Correção ambiental dentro do experimento}

A correção dos dados para efeito ambiental foi feita, utilizando-se como fator de correção a variação nos valores de cada cultivar-padrão em relação à média geral dos cultivares-padrão para dada característica. A matriz composta pelos fatores de correção, 
para cada característica, foi obtida da seguinte forma: obteve-se a média geral, em relação a cada cultivarpadrão; obteve-se a média de cada repetição em cada cultivar-padrão; o fator de correção foi obtido pela subtração da média da repetição do cultivar-padrão 1, ou do cultivar-padrão 2 , pela correspondente média geral e cada valor fenotípico dos indivíduos segregantes foi subtraído deste fator, obtendo assim o valor corrigido.

O número de linhas da matriz de correção foi igual à soma do número de repetições dos cultivares-padrão. A correção foi feita de acordo com a seguinte expressão: $V_{C_{i j}}=V_{o_{i j}-} F_{C_{z k}}$, em que: valor corrigido para o indivíduo j dentro da linha segregante $\mathrm{i} ; V_{o_{i j}}$ : valor observado do indivíduo $\mathrm{j}$ na linha i; e $F_{C: x}$ : fator de correção originado do cultivarpadrão $\mathrm{z}$, na repetição $\mathrm{k}$.

\section{Seleção}

As estratégias de seleção utilizadas nas populações $(\mathrm{F})$ dos três cruzamentos e o número de indivíduos selecionados em cada uma delas foram os seguintes: i) Seleção entre e dentro de famílias para produção de grãos: os percentuais de famílias selecionadas de acordo com o cruzamento corresponderam aos seguintes: $\mathrm{CR} 1=26,4 \%, \mathrm{CR} 2=$ $35,8 \%$ e CR3 $=44,5 \%$; foram selecionados $33,4 \%$ dos melhores indivíduos de cada família superior. Assim, selecionaram-se 40 indivíduos por cruzamento, que foi fixado para as demais estratégias. ii) Seleção combinada: foram selecionados os 40 indivíduos que apresentaram os maiores valores genéticos obtidos da combinação entre o desvio do indivíduo em relação à média da família e o desvio da média da família em relação à média geral para produção de grãos. iii) Seleção individual para produção de grãos: 40 indivíduos foram selecionados, desconsiderando-se a informação da família, ou seja, selecionaram-se os 40 indivíduos mais produtivos. iv) Seleção livre de pesos de parâmetros: foram selecionados 40 indivíduos, considerando-se número de dias para maturação, altura de planta na maturação e produção de grãos como características da seleção simultânea. Os limites inferiores de seleção corresponderam a: 125 dias para maturação, 65 centímetros para altura da planta e média da produção de grãos para cada cruzamento.

Os 40 indivíduos selecionados por estratégia de seleção corresponderam a diferentes porcentagens de seleção, devido ao tamanho diferenciado das populações. Esse número de indivíduos selecionados corresponde às seguintes percentagens de seleção por cruzamento: CEPS 77-16 x Doko RC: 8,77\%, CEPS 89-26 x IAC-8: 11,90\% e CEPS 89-26 x FT-Cristali- na: $14,81 \%$. Houve, portanto, sobreposição de indivíduos nas diferentes estratégias de seleção, o que resultou numa população selecionada com os seguintes números de indivíduos: CR1: 84, CR2: 81 e CR3: 75. A seleção foi feita com base nos dados corrigidos para efeito ambiental.

\section{Estimativas das variâncias genéticas e das herdabilidades em sentido restrito}

As estimativas das variâncias genéticas e das herdabilidades em sentido restrito estão apresentadas na tabela 1 .

\section{Predição de ganhos \\ Seleção individual}

A resposta à seleção individual foi estimada, utilizando-se a expressão apresentada na tabela 1 .

Índice de seleção livre de pesos e de parâmetros A seleção dos indivíduos utilizando o índice de seleção livre de pesos e de parâmetros foi feita, mediante o emprego da expressão, conforme descrita por ELSTON (1963), que está apresentada na tabela 1. Após selecionados os indivíduos, utilizando-se as características número de dias para maturação, altura da planta na maturação e produção de grãos para compor o índice de seleção. a resposta à seleção foi estimada para cada característica isoladamente, utilizando-se procedimento semelhante à seleção individual, ou seja: $G S_{X}=D S_{X} \times h_{r X}^{2}$

\section{Seleção entre e dentro de famílias}

Segundo VENCOVSKY (1987), uma vez definidos as famílias e os indivíduos dentro das famílias que comporão a população melhorada, o ganho por seleção pode ser estimado conforme expressão apresentada na tabela 1 .

\section{Seleção combinada}

A seleção foi feita a partir de um índice, o qual foi obtido, levando-se em conta o desvio do indivíduo em relação à média da família à qual pertencia e o desvio da média da família em relação à média geral. Para cada desvio foi dado um peso, objetivando minimizar as diferenças entre os elementos do vetor I, de índices de seleção, e os elementos correspondentes do vetor $\mathrm{G}$, de valores genéticos. A expressão para o índice de seleção combinada está apresentada na tabela 1, sendo os pesos estimados a partir de: $b_{1}=\left(1-r_{g w}\right) h_{R w}^{2}$ e, $b_{2}=\left(\frac{1-r_{g w}}{p}+r_{g w}\right) h_{R F}^{2}$ 
Tabela 1 - Estimadores dos parâmetros utilizados para obtenção das variâncias, herdabilidades e ganhos através dos diferentes procedimentos de seleção.

\begin{tabular}{ll}
\hline Parâmetros & Estimadores \\
\hline Variância genética entre médias de famílias & $\hat{\sigma}_{G e}^{2}=\hat{\sigma}_{F e}^{2}-\hat{\sigma}_{e}^{2}$ \\
Variância genética dentro de famílias & $\hat{\sigma}_{G w}^{2}=\hat{\sigma}_{F w}^{2}-\hat{\sigma}_{e w}^{2}$ \\
Variância aditiva & $\hat{\sigma}_{A}^{2}=\frac{\hat{\sigma}_{G e}^{2}+I_{n} \hat{\sigma}_{G w}^{2}}{I_{n}\left(1+I_{n}\right)}$ \\
Herdabilidade entre famílias & $h_{\mathrm{Re}}^{2}=\frac{2 I_{n} \hat{\sigma}_{A}^{2}}{\hat{\sigma}_{F e}^{2}}$ \\
Herdabilidade dentro de famílias & $h_{R w}^{2}=\frac{\left(1-I_{n}\right) \hat{\sigma}_{A}^{2}}{\hat{\sigma}_{F w}^{2}}$ \\
Herdabilidade em nível de indivíduo & $h_{R I}^{2}=\frac{\left(1+I_{n}\right) \hat{\sigma}_{A}^{2}}{\hat{\sigma}_{F_{\text {Total }}}^{2}}$ \\
Ganho de seleção individual & $G S_{X}=D S_{X} \times h_{r X}^{2}$ \\
Ganho de seleção entre e dentro & $G S=\frac{\hat{\sigma}_{G e}^{2}}{\hat{\sigma}_{F e}^{2}} D S_{1}+\frac{\hat{\sigma}_{G w}^{2}}{\hat{\sigma}_{F w}^{2}} D S_{2}$ \\
Índice de seleção livre de pesos e de parâmetros & $I_{e}=\prod_{j=1}^{n} w_{j}$ \\
Índice de seleção combinada & $I_{i j}=b_{1}\left(Y_{i j}-\bar{Y}_{i .}\right)+b_{2}\left(\bar{Y}_{i .}-\bar{Y} ..\right)$ \\
\hline
\end{tabular}

$\hat{\sigma}_{F e}^{2}$ : estimativa da variância fenotípica entre médias de famílias; $\hat{\sigma}_{F w}^{2}$ : estimativa da variância fenotípica dentro de famílias; $\hat{\sigma}_{e}^{2}$ : estimativa da variância ambiental entre médias de famílias; $\hat{\sigma}_{e w}^{2}$ : estimativa da variância ambiental dentro de famílias; $I_{n}$ : coeficiente de endogamia na geração considerada; $D S_{X}$ : diferencial de seleção do caráter $\mathrm{X} ; h_{r X}^{2}$ : herdabilidade, no sentido restrito, do caráter $\mathrm{X}$, em nível de indivíduo; $D S_{1}$ : diferencial médio de seleção para família; $D S_{2}$ : diferencial de seleção médio de indivíduo dentro de família; $b_{1}:$ peso dado ao desvio do valor do indivíduo $\left(Y_{i j}\right)$ em relação à media de sua família $\bar{Y}_{i}$. $) ; b_{2}$ : peso dado ao desvio da média da família $\left(\bar{Y}_{i}\right.$. ) em relação à média geral da população $(\bar{Y}$.. ).

em que: $r_{g w}$ correlação entre plantas dentro de parcelas ou famílias; p: número de plantas avaliadas por parcela ou família.

A resposta à seleção é dada pelo diferencial de seleção no índice: $R S=D S_{I}$

Em todas as estratégias, os ganhos esperados em virtude da seleção foram obtidos de dados corrigidos. Foram também estimados os ganhos esperados de dados originais com a seleção feita nos dados corrigidos.

\section{RESULTADOS E DISCUSSÃO}

\section{Seleção entre e dentro de famílias para produção} de grãos

Na estimação dos parâmetros para dados corrigidos, apenas a variância fenotípica foi alterada, sendo as demais variâncias as mesmas estimadas para dados originais (Tabela 2).

Nas estimativas referentes ao cruzamento CR1, notou-se que a correção dos dados diminuiu a variação fenotípica com referência à variação entre mé- 
Tabela 2 - Estimativas de parâmetros genéticos para dados originais e corrigidos para efeito ambiental em produção de grãos utilizando-se seleção entre e dentro de famílias e seleção combinada nos três cruzamentos (CR1, CR2 e CR3) em progênies $\mathrm{F}_{5}$ de soja $^{1}$

\begin{tabular}{|c|c|c|c|c|c|c|}
\hline \multirow{2}{*}{ Parâmetros ${ }^{2}$} & \multicolumn{2}{|c|}{ CR1 } & \multicolumn{2}{|c|}{ CR2 } & \multicolumn{2}{|c|}{ CR3 } \\
\hline & Entre famílias & $\begin{array}{l}\text { Dentro de } \\
\text { famílias }\end{array}$ & Entre famílias & $\begin{array}{l}\text { Dentro de } \\
\text { famílias }\end{array}$ & Entre famílias & $\begin{array}{l}\text { Dentro de } \\
\text { famílias }\end{array}$ \\
\hline$h_{R}^{2}$ & 0,523 & 0,126 & 0,258 & 0,053 & 0,770 & 0,377 \\
\hline$h_{R C}^{2}$ & 0,634 & 0,126 & 0,192 & 0,053 & 0,844 & 0,377 \\
\hline $\bar{X}_{O}$ & 12,545 & 12,545 & 9,337 & 9,337 & 14,404 & 14,404 \\
\hline $\bar{X}_{S}$ & 17,339 & 25,565 & 12,498 & 14,804 & 18,212 & 25,022 \\
\hline $\bar{X}_{S C}$ & 16,250 & 24,476 & 12,886 & 14,407 & 18,765 & 25,576 \\
\hline$G S \%$ & 19,976 & 13,033 & 8,739 & 3,126 & 20,367 & 27,820 \\
\hline$G S_{C} \%$ & 18,722 & 11,941 & 7,308 & 2,910 & 25,554 & 29,275 \\
\hline$\hat{b}_{1}=\hat{b}_{1 c}$ & \multicolumn{2}{|c|}{1,000} & \multicolumn{2}{|c|}{1,000} & \multicolumn{2}{|c|}{1,000} \\
\hline$\hat{b}_{2}$ & \multicolumn{2}{|c|}{4,163} & \multicolumn{2}{|c|}{4,831} & \multicolumn{2}{|c|}{2,041} \\
\hline$\hat{b}_{2 C}$ & \multicolumn{2}{|c|}{5,047} & \multicolumn{2}{|c|}{3,588} & \multicolumn{2}{|c|}{2,236} \\
\hline$G S C \%$ & \multicolumn{2}{|c|}{41,031} & \multicolumn{2}{|c|}{14,298} & \multicolumn{2}{|c|}{72,889} \\
\hline$G S C_{C} \%$ & \multicolumn{2}{|c|}{31,757} & \multicolumn{2}{|c|}{11,085} & \multicolumn{2}{|c|}{74,083} \\
\hline$G S C_{C} / G S C$ & \multicolumn{2}{|c|}{0,773} & \multicolumn{2}{|c|}{0,775} & \multicolumn{2}{|c|}{1,016} \\
\hline$E F S C$ / SED & \multicolumn{2}{|c|}{1,035} & \multicolumn{2}{|c|}{1,085} & \multicolumn{2}{|c|}{1,351} \\
\hline$E F S C_{c o r} / S E D_{c o r}$ & \multicolumn{2}{|c|}{1,264} & \multicolumn{2}{|c|}{1,235} & \multicolumn{2}{|c|}{1,352} \\
\hline
\end{tabular}

${ }^{1}$ CR1: 'CEPS 77-16’ X 'Doko RC' ; CR2: ‘CEPS 89-26’ X 'IAC - 8' ; CR3: ‘CEPS 89-26’ x 'FT-Cristalina'.

${ }^{2}$ herdabilidade restrita $\left(h_{R}^{2}\right)$; herdabilidade restrita para dados corrigidos pelo efeito ambiental $\left(h_{R C}^{2}\right)$; média original ( $\left.\bar{X}_{O}\right)$; média dos selecionados com base no valor fenotípico $\left(\bar{X}_{S}\right)$; média dos selecionados com base no valor corrigido para efeito ambiental $\left(\bar{X}_{S C}\right)$; ganhos percentuais $(G S \%)$ e $\left(G S_{C} \%\right)$; pesos dados ao desvio do indivíduo em relação à média da família para dados originais e corrigidos $\left(\hat{b}_{1} e \hat{b}_{1 c}\right)$; peso dado ao desvio da média da família em relação à média geral para dados originais $\left(\hat{b}_{2}\right)$ e para dados corrigidos para efeito ambiental $\left(\hat{b}_{2 C}\right)$; ganhos percentuais pela seleção combinada $\left(G S C \%\right.$ ) e $\left(G S C_{C} \%\right.$ ); relação dos ganhos para dados corrigidos e fenotípicos $\left(G S C_{C} / G S C\right)$; eficiência da seleção combinada sobre seleção entre e dentro de famílias $(E F . S C / S E D)$ e eficiência da seleção combinada para dados corrigidos sobre seleção entre e dentro de famílias para dados corrigidos ( $E F S C_{c o r} / S E D_{c o r}$ ).

dias de famílias, o que refletiu numa expectativa de maior representatividade do valor genético pelo valor corrigido através do fator de correção ambiental. Isso indicou que a correção influen- ciou de maneira a reduzir aqueles valores fenotípicos que foram beneficiados e aumentar aqueles valores prejudicados pelo efeito não-genético, gerando, com isso, maior expectativa de 
eficiência quanto ao ganho real comparado ao valor fenotípico original.

No cruzamento CR2, a correção dos dados aumentou a variação total ou fenotípica em nível de média de família, o que acarretou redução da herdabilidade nesta unidade de seleção. Isso pode ser devido a uma correção que não refletia a variação nãogenética, a não ser que existisse tendência de os genótipos superiores estarem em locais desfavoráveis ou os genótipos inferiores estarem em locais de influência ambiental favorável. Já no cruzamento CR3, mostraram-se valores altos de herdabilidade, tanto em nível de médias de famílias quanto de indivíduos dentro de famílias, para dados fenotípicos originais e corrigidos. Observou-se também que esse foi o cruzamento mais promissor nessa estratégia, pois foi o que apresentou maior expectativa de ganho, em comparação com os demais. Além disso, foi nesse cruzamento que se selecionou a maior proporção de indivíduos em relação à população original, devido ao fato de ser esta a população mais reduzida entre as três e de o número de indivíduos selecionados manter-se constante para cada estratégia.

Numa comparação geral entre os três cruzamentos, notou-se que a correção dos dados não afetou a variabilidade dentro de famílias, o que refletiu no mesmo coeficiente de herdabilidade para dados corrigidos e não-corrigidos. Houve expectativa de maior ganho no cruzamento CR3, que foi em função da maior estimativa de herdabilidade, tanto entre famílias quanto dentro de famílias, com ou sem correção dos dados. Logo, a eficiência da predição para essa estratégia quanto ao comportamento no campo, para CR3, ficou em função da precisão das estimativas das herdabilidades.

\section{Seleção combinada}

As estimativas dos coeficientes para o desvio do indivíduo em relação à média da família e o desvio da média da família em relação à média geral, para dados originais e corrigidos pelo efeito ambiental assim como as expectativas de ganhos para os três cruzamentos em virtude da seleção combinada univariada para produção de grãos estão apresentados na tabela 2. Em trabalhos com esse índice de seleção combinado, foi verificada variação em relação à seleção entre e dentro convencional, pelo fato de o índice considerar o valor individual e as médias das famílias de maneira ponderada, o que resultou na seleção de um número diferenciado de famílias e de indivíduos por família, enquanto na seleção entre e dentro convencional, após a seleção da família, o número de in- divíduos permanece constante por família (PIRES, 1996). Um risco a que o melhorista está sujeito quando usa esse tipo de índice de seleção combinada é, conforme relataram VIANA e CRUZ (1997), selecionar plantas excepcionais, cuja superioridade seja devido a fatores não genéticos, as quais pertençam a famílias com desempenho inferior em detrimento de plantas boas pertencentes a famílias de comportamento superior.

As estimativas de progressos genéticos advindos da seleção combinada é variável entre os cruzamentos e apresentam magnitudes muito expressivas. Os pesos $\hat{b} 1, b 2$ e $b 2 c$, na forma de pesos ponderados para herdabilidade restrita dentro de famílias, indicaram a importância relativa de cada desvio na composição do índice que expressa o valor genético da planta. Essa ponderação, pode refletir em maior ou menor indicativo de presença de variância ambiental entre e dentro de famílias (VIANA e CRUZ, 1997). Com isso, quanto maior o índice ponderado, maior será o efeito ambiental dentro de família, sendo também esse índice associado ao coeficiente gerado pela porção da variância genética aditiva explorada entre famílias em relação à porção explorada dentro de famílias, ocorrendo decréscimo desta última parte, geração após geração, em plantas de autopolinização. A expectativa de maior ganho com a utilização da seleção combinada está no cruzamento que apresenta a menor relação $b_{2} / b_{1}$ e $b_{2 c} / b_{1}$

A eficiência da seleção combinada, em relação à seleção entre e dentro, evidenciou valores da ordem de 1,035 a 1,352, variando de cruzamento para cruzamento, e correção ou não dos dados. No entanto, com a correção dos dados, nota-se maior expectativa de eficiência da seleção combinada em relação à seleção entre e dentro, superando valores citados por FALCONER (1987), que são de até $10 \%$.

\section{Seleção individual para produção de grãos}

As expectativas de ganhos nos caracteres avaliados em virtude da seleção em produção de grãos foram estimadas para que se pudesse prever seu comportamento e comparar à seleção simultânea (Tabela 3). A seleção em produção de grãos previa ganhos indiretos tanto em número de dias para maturação, quanto em altura de planta na maturação, nos três cruzamentos, para dados originais e corrigidos. Esses ganhos indiretos foram de magnitudes relativamente baixas, o que está associado a um reduzido diferencial de seleção indireto nestes caracteres, uma vez que as herdabilidades para os dois caracteres, nos três cruzamentos, foram bastante expressivas. Quanto à produção de grãos, a expectativa de maiores ganhos está na 
Tabela 3 - Estimativas de parâmetros genéticos para dados originais e corrigidos para efeito ambiental em produção de grãos, número de dias para maturação e altura de planta na maturação utilizando-se seleção individual e a seleção "livre de pesos" e "livre de parâmetros" nos três cruzamentos (CR1, CR2 e CR3) em progênies $F_{5}$ de soja ${ }^{1}$

\begin{tabular}{|c|c|c|c|c|c|c|c|c|c|}
\hline \multirow{2}{*}{ Parâmetros ${ }^{2}$} & \multicolumn{3}{|c|}{ CRI } & \multicolumn{3}{|c|}{$\mathrm{CR} 2$} & \multicolumn{3}{|c|}{ CR3 } \\
\hline & NDM & APM & PRO & NDM & APM & $\mathrm{PRO}$ & NDM & APM & PRO \\
\hline$h_{R}^{2}$ & 0.999 & 0,880 & 0.432 & 1,000 & 0.899 & 0,206 & 0.871 & 0,813 & 0.720 \\
\hline$h_{R C}^{2}$ & 1.000 & 0.861 & 0.499 & 0,824 & 0.402 & 0.164 & 0.730 & 0.752 & 0,780 \\
\hline $\bar{X}_{0}$ & 135.239 & 63,274 & 12,545 & 131.065 & 67.929 & 9,337 & 132.100 & 58,930 & 14,404 \\
\hline $\bar{X}_{S}$ & 137.725 & 70.275 & 30,204 & 133,925 & 70.400 & 18,605 & 135.475 & 66.100 & 30,501 \\
\hline $\bar{X}_{i c}$ & 137.126 & 72.446 & 28.264 & 134.100 & 68.958 & 18.887 & 136.120 & 66.682 & 29,704 \\
\hline$G S \%$ & 1.836 & 9.742 & 60.768 & 2.182 & 3,272 & 20,398 & 2.225 & 9.898 & 80.507 \\
\hline$G S_{i} \%$ & 1.395 & 12,483 & 62.549 & 1.908 & 0.609 & 16.772 & 2.233 & 9,895 & 82,828 \\
\hline $\bar{X}_{S i}$ & 139,450 & 87.725 & 21.392 & 138,775 & 89,250 & 15,176 & 136,950 & 73,150 & 22.806 \\
\hline $\bar{X}_{\mathrm{N} /}$ & 139.006 & 88.581 & 18.061 & 138,725 & 91.154 & 15,356 & 137,570 & 75,124 & 23,784 \\
\hline$G S I \%$ & 3,111 & 34,025 & 30.440 & 5,882 & 28,230 & 12,851 & 3.197 & 19,630 & 42,022 \\
\hline$G S I_{c} \%$ & 2.785 & 34,441 & 21.949 & 4,815 & 13,740 & 10,571 & 3.025 & 20.671 & 50,779 \\
\hline
\end{tabular}

'CR1: 'CEPS 77-16` X'Doko RC' : CR2: 'CEPS 89-26’ X'IAC-8’ CR3: 'CEPS 89-26’ x 'FT-Cristalina'.

${ }^{2}$ herdabilidades restritas em nivel de individuo para dados originais $\left(h_{R}^{2}\right)$ e para dados corrigidos $\left(h_{R C}^{2}\right)$ : médias originais $\left(\bar{X}_{0}\right)$ : médias fenotipicas dos individuos selecionados $\left(\bar{X}_{S}\right)$ : médias dos individuos selecionados com base nos dados corrigidos para efeito ambiental $\left(\bar{X}_{S}\right)$ : ganhos percentuais com base no valor fenotipico (GS \%) e com base no valor corrigido (GSc \%) para seleção individual: médias fenotipicas dos individuos selecionados $\left(\bar{X}_{S /}\right)$ e médias dos individuos selecionados com base nos dados corrigidos para efeito ambiental $\left(\bar{X}_{S / K^{\circ}}\right.$ ) para seleção "livre de pesos". e "livre de parâmetros": ganho, em porcentagem, da média original esperada pela seleçào com base no valor fenotipico e no valor corrigido ( $G S I \%$ ) e ( $G S I, \%$ ), respectivamente, utilizando-se a seleção "livre de pesos" e "livre de parâmetros".

população pertencente ao cruzamento CR3, que apresenta os maiores coeficientes de herdabilidade entre os três cruzamentos, associado ao maior diferencial de seleção, o que permite afirmar que é uma população promissora, por apresentar elevada variabilidade genética. Já o cruzamento CR1 apresenta elevado diferencial de seleção, mas reduzida representatividade do valor genético pelo fenotípico.

\section{Seleção livre de pesos e de parâmetros}

Em todos os cruzamentos houve ganhos em número de dias para maturação, altura da planta na maturação e produção de grãos, sendo portanto o mais promissor, com referência à produção de grãos, o cruzamento CR3 - CEPS 89-26 x FT-Cristalina (Tabela 3). A variação no diferencial de seleção, quando baseada em dados corrigidos pela variação ambiental e dados fenotípicos, foi pequena, evidenciando que o fator de correção foi aleatório nas progênies selecionadas, caso contrário poderia ter subestimado ou superestimado determinadas progênies numa determinada faixa do experimento.

Comparando estes resultados com a seleção individual, a expectativa de ganhos em produção de grãos foi menor em todos os cruzamentos. No entanto, para os caracteres número de dias para maturação e altura da planta na maturação, também considerados na seleção, o ganho foi maior. Os caracteres número de dias para maturação e altura da planta na maturação, em soja, apresentaram, além de alta herdabilidade, alta associação genética com o caráter produção de grãos, podendo ser importante no processo seletivo (JOHNSON et al., 1955; KNOW \& TORRIE, 1964; SANTOS, 1994). 


\section{CONCLUSÕES}

A seleção combinada é superior à seleção entre e dentro de famílias, tanto para dados corrigidos quanto para os não corrigidos; a estratégia seleção individual promove maior expectativa de ganho genético para produção de grãos; o cruzamento de maior expectativa de ganhos em produção de grãos em todas as estratégias de seleção é o CR3; o método de correção dos dados pela variação de linhagens homozigóticas (cultivares-padrão) é uma alternativa para o melhorista de plantas como auxílio no processo seletivo.

\section{REFERÊNCIAS BIBLIOGRÁFICAS}

CRUZ, C.D.; REGAZZI, A.J. Modelos biométricos aplicados ao melhoramento genético. Viçosa : UFV, 1997. 390p.

ELSTON, R.C. A weigth-free index for the purpose of ranking or selection with respect to several traits at a time. Biometrics, v.19, n.1, p.85-97, 1963.

FALCONER, D.S. Introdução à genética quantitativa. Viçosa: UFV, 1987. 279p.

HALLAUER, A.R.; MIRANDA FILHO, J.B. Quantitative genetics in maize breeding. Ames : Iowa State University, 1981. $468 \mathrm{p}$.

JENNINGS, P.R.; HERRARA, P.M. Studies on competition in rice. II. Competition in segregating populations. Evolution, v.22, n.2, p.332-336, 1968.

JOHNSON, H.W.; ROBINSON, H.F.; COMSTOCK, R.E. Genotipic and phenotypic correlations in soybeans and their implications in selection. Agronomy Journal, Madison, v.47, n.10, p.477-483, 1955.

KHALIFA, M.A.; QUALSET, C.O. Intergenotypic competition between tall and dwarf wheats. II. In hybrid bulks. Crop Science, Madison, v.15, n.5, p.640-644, 1975.

KNOW, S.H.; TORRIE, J.H. Heritability of and interrelationships among traits of two soybean populations. Crop Science, v.4, n.2, p.196-198, 1964.

LUSH, J.L. Melhoramento dos animais domésticos. Rio de Janeiro : CEDEGRA, 1964. 566p.

PIRES, I.E. Eficiência da seleção combinada no melhoramento genético de Eucalyptus spp. 1996. 116f. Tese (Doutorado em Genética e Melhoramento) - Universidade Federal de Viçosa.

RAMALHO, M.A.P.; VENCOVSKY, R. Estimação dos componentes da variância genética em plantas autógamas. Ciência e Prática, Lavras, v.2, n.2, p.117-140, 1978.

SAnTOS, C.A.F. Análise de trilha e estimativas de parâmetros genéticos em progênies $F_{6}$ de um cruzamento de soja (Glycine max (L.) Merrill). 1994. 71f. Dissertação (Mestrado em Genética e Melhoramento) - Universidade Federal de Viçosa.

VENCOVSKY, R. Herança quantitativa. In: PATERNIANI, E.; VIEGAS, G.P. Melhoramento e produção do milho no Brasil. 2.ed. Campinas : Fundação Cargill, 1987. p.137-214.

VIANA, J.M.S.; CRUZ, C.D. Combined selection in early generation testing of self-pollinated plants. Brazilian Journal of Genetics, Ribeirão Preto, v.20, n.4, p.673-681, 1997.

WEBER, W.E. Selection in segregating of autogamous species. I. Selection response for combined selection. Euphytica, Wageningen, v.31, n.2, p.493-502, 1982. 